\title{
Adaptive Feature Transformation for Classification with Sparse Representation
}

\author{
Yaxin Sun, Guihua Wen
}

\begin{abstract}
Sparse representation based classification (SRC) has been shown to be an effective method for face recognition. Furthermore, the input features of more and more classifiers are extracted by dimensional reduction methods. However, we find that the reconstruction ability of a basis for a testing sample is related with cosine distance between this basis and this testing sample, but most dimensional reduction methods are based on Euclidean distance. Obviously, a gap is existed between dimensional reduction methods and SRC. In this paper, we propose an adaptive feature transformation based on self-tuning point to point distances (SPPDAFT) to transform features to a new feature space. The SPPDAFT can makes that the cosine distances among samples in new feature space increase with the Euclidean distances among samples in original space. As a result, the reconstruction ability of a basis to a testing sample in new feature space would be indirectly related with the Euclidean distance between this basis and this sample in original space, and then the gap between dimensional reduction methods and SRC can be reduced. The experimental results on benchmark databases show the effectiveness of SPPDAFT.
\end{abstract}

Index Terms-Sparse Representation Classification, Dimensional Reduction, Feature Transformation, Face Recognition

\section{Introduction}

Sparse representation has recently been applied to a variety of applications in computer vision and machine learning [1-5]. Its success is attributed to the fact that the dimensionality of signals such as natural images is often much lower than that which is observed, and thus it offers a more compact yet better description of natural signals for the above applications[4]. To extend sparse representation to the problems of classification, Wright [36] proposed sparse representation based classification (SRC), which obtains good results on face recognition.

However, there are three defects for SRC. Firstly, due to the over complete codebook and the independent coding process, the locality and the similarity among the samples are lost [17 23]. Secondly, using original training samples as the dictionary cannot fully exploit the discriminative information hidden in the training samples [9-13]. Thirdly, dimensional reduction methods are often used to extracted features for SRC $[8,11,26,27,30,36]$, however, a gap is existed between these dimensional reduction methods and SRC, because the goal of most dimensional reduction methods is maximizing the Euclidean distances across classes and minimizing the

The authors are with the School of Computer Science and Engineering, South China University of Technology, Guangzhou 510006, China. E-mail: sunyaxin2005@163.com, crghwen@scut.edu.cn
Euclidean distances within the class, while the reconstruction ability of each basis for a testing sample is related on the cosine distances between this basis and this sample.

To overcome the first defect, some optimization problems for sparsely coding have been proposed [14,17-18, 20-23]. These methods think that the locality among the coefficients of samples should be the same as the locality among these samples, and add an item in optimization problems to keep above relationship. Obviously, the reconstruction ability of each basis does not be changed.

To overcome the second defect, some dictionary learning methods have been proposed. Jiang[9] introduces a new label consistent KSVD (LCKSVD), which learns a single over complete dictionary, a linear transformation matrix, and an optimal linear classifier jointly. However, the transformation matrix is learned for sparse coefficients but not for the bases, so that the reconstruction ability of each basis does not be changed. Chen [12] proposes a discriminative low-rank representation (DLRR), which makes the dictionary elements between different classes as independent as possible. However, DLRR minimizes the reconstruction errors between dictionary and training data, and then the variances of the reconstruction abilities of bases are very small. Yang [13] proposed a fisher discrimination dictionary learning (FDDL) approach for sparse representation classification. However, the test samples without any transformation, and then the learned dictionary may be not suitable to testing samples.

To overcome the third defect, three methods can be used: (1) the input features of SRC can be mapped to a kernel space [6, 7], however, a suitable kernel is hardly chosen. (2) Setting different weights for the sparse coefficients [15, 19, 26-28], however, the reconstruction abilities of bases also does not be changed. Thirdly, using sparse representation based dimensional reduction methods [16,29-32]. Can-Yi Lu [29] proposed optimized projections for SRC (OP-SRC), it aims to reduce the within-class reconstruction residual and increase the across-class reconstruction residual, however, the reconstruction residual are computed from high dimensional space, and then the reconstruction residual would not be keep in low dimensional space. Jie Gui[30] proposed discriminant sparse neighborhood preserving embedding (DSNPE), it preserves the sparse reconstructive relationship among the samples and sufficiently utilizes the global discriminant structures, however, the define of discriminant structures is also based on Euclidean distances, and then a gap is also existed.

In this paper, we propose an adaptive feature transformation 
based on self-tuning point to point distances (SPPDAFT) to overcome the third defect. SPPDAFT transforms the features to a new feature space, and makes that the cosine distances among samples in new feature space increase with the Euclidean distances among samples in original space. As a result, the reconstruction ability of a basis to a testing sample in new feature space would relate with the Euclidean distance between this basis and this sample in original space, and then the SRC can fully utilize the information extracted by dimensional reductions methods, which means that the third defect of SRC can be overcome. Obviously, if the features extracted by dimensional reduction contain the discriminative information, the dictionary in new feature space also contains discriminative information, and then the second defect of SRC can be overcome. Furthermore, because the reconstruction ability of a basis to a testing sample relate with the cosine distance between this basis and this testing sample, so that two testing samples with a small cosine distance would have similar cosine relationships with bases, and then the first defect of SRC is also overcome.

The remainder of this paper is organized as follows. Section 2 introduces the related work. Section 3 introduces the proposed SPPDAFT and describes how SPPDAFT performed with SRC. Experimental results are presented and discussed in Section 4. Finally, Section 5 gives concluding remarks.

\section{Related Work}

The proposed SPPDAFT is used to reduce the gap between the supervised or semi-supervised dimensional reduction and SRC, so that in this section we first review SRC, and then review supervised or semi-supervised dimensional reduction.

\section{A. Sparse representation based classification (SRC)}

Wright et al [36] proposed the sparse representation based classification (SRC) for robust face recognition. Suppose that we have $c$ classes, and let $D=\left[D_{1}, D_{2}, \cdots, D_{c}\right]$ be the set of original training samples, where $D_{c}$ is the sub-set of the training samples from class $c$. Denote by $x$ a testing sample. The procedures of SRC are as follows.

(1) Sparsely code $x$ on $D$ via $l_{1}$-norm minimization

$$
\hat{\alpha}=\arg \min _{\alpha}\|x-D a\|_{2}^{2}+\lambda\|\alpha\|_{1}
$$

Where $\lambda$ is a scalar constant, $\|x-D a\|_{2}^{2}$ is used to computed the reconstruction error, and $\|\alpha\|_{1}$ is used to keep sparse of $a$

(2) Compute reconstruction error for each class

$$
E_{c}=\left\|x-D_{c} \hat{\alpha} c\right\|_{2}^{2}
$$

(3) Do classification via

$$
\hat{c} \hat{c}=\operatorname{SRC}(x)=\arg \min _{c} E_{c}
$$

\section{B. Kernel Sparse representation based classification (KSRC)}

Suppose that we have the training samples $X=\left[x_{1}, x_{2}, \cdots x_{n}\right]$ and a testing sample $x$, the procedures of KSRC are as follows.

(1) Select a Mercer kernel $k(\cdot, \cdot)$. Where the linear kernel is presented by Eq.(4) and the RBF kernel is presented by Eq.(5), where $\gamma$ is the parameter of RBF kernel.

$$
\begin{aligned}
& k\left(x_{i}, x_{j}\right)=x_{i}{ }^{T} x_{j} \\
& k\left(x_{i}, x_{j}\right)=\exp \left(-\gamma\left\|x_{i}-x_{j}\right\|_{2}^{2}\right)
\end{aligned}
$$

(2) Compute the kernel Gram matrix $K$, where $K_{i j}=k\left(x_{i}, x_{j}\right)$, and $k(\cdot, x)=\left[k\left(x_{1}, x\right), \cdots, k\left(x_{n}, x\right)\right]^{T}$.

(3) Normalize the columns of $K$ and $k(\cdot, x)$ to have unit $l_{2}$-norm.

(4) Set $D=K, x=k(\cdot, x)$, and use Eq.(1) to (3) to get the class label of $x$.

\section{Supervised or semi-supervised dimensional reduction}

Recently, a large number of dimensional reduction methods can be used to map features from high dimensional space to low dimensional space, such as: Principal Component Analysis (PCA) [45], Linear Discriminant Analysis (LDA) [45], Neighborhood Preserving Embedding (NPE) [37], and some semi-supervised dimension reduction methods [38-44]. Most of supervised or semi-supervised dimension reduction methods have the following framework:

$$
W^{*}=\underset{W^{T} W=I}{\arg \max } \frac{\lambda_{1} W^{T} S_{b} W+\lambda_{2} W^{T} S_{f} W}{\lambda_{3} W^{T} S w W+\lambda_{4} W^{T} S_{m} W}
$$

Where $W$ is a mapping matrix, which is used to map the feature from high dimensional space to low dimensional space; $S_{b}, S_{w}, S_{f}, L^{M}$ are used to define the relationships among training points; and $\lambda_{1}, \lambda_{2}, \lambda_{3}, \lambda_{4}$ are scalar constants.

From the principle of most supervised or semi-supervised dimensional reduction [37-45], constructing $S_{b}, S_{w}, S_{f}, L^{M}$ is based on Euclidean distance, so that the classifier that can utilize the advantage of these supervised or semi-dimensional reduction methods should be also based on Euclidean distance. However, in SRC, the reconstruction ability of bases are not changed with Euclidean distances.

\section{Adaptive Feature Transformation for Classification with Sparse Representation}

We now detail our proposed algorithm. In the first subsection, we will introduce the motive of SPPDAFT. In the second subsection, we will give the proposed SPPDAFT. In the third subsection, we will give how SPPDAFT is used with SRC. And in the last subsection, we compare the SPPDAFT with some related algorithms.

\section{A. Motive}

Many algorithms can be used to solve $l_{1}$-norm minimization problem, such as: Least Angle Regression (LARs) [33], Gradient Projection for Sparse Reconstruction (GPSR) [34], and so on. From these algorithms, we find that the reconstruction ability of a basis to a testing sample is greatly influenced by the cosine distance between this basis and this sample. However, the goal of most dimensional reduction methods is maximizing the Euclidean distances across classes and minimizing the Euclidean distances within class. As a result, a gap is existed between SRC and most dimensional reduction methods, which can be shown by an example. The example is presented on Fig.1. 
(a)

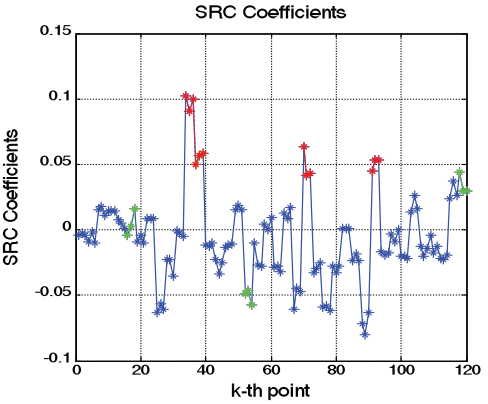

(b)

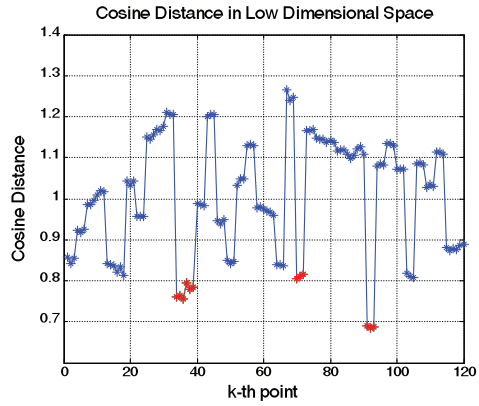

(c)

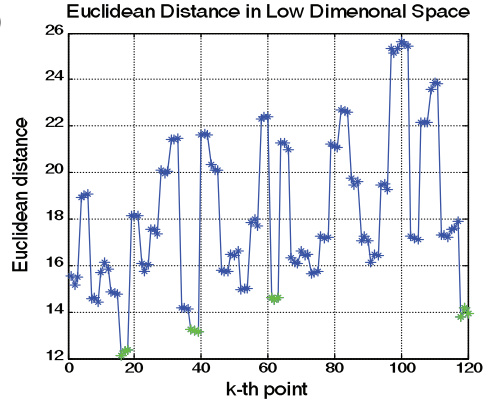

Fig.1 An example used to show the gap between dimensional reduction and SRC. (a) Sparse coefficients of bases to a testing sample, (b) cosine distances

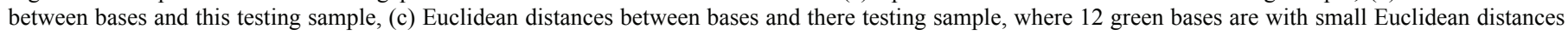
to the testing sample, and 12 red bases are with samll cosine distances to the testing sample.

It can be seen from Fig. 1 that the coefficients of 12 red bases are all very large, but most of coefficients of 12 green bases are very small, obviously, the reconstruction ability of bases in this example are more related with cosine distances than with Euclidean distances.

Some people think that normalized the samples with length 1, the cosine distances can increase with Euclidean distances, and then the gap can be eliminated. However, we all know that normalized the samples with length 1 not changes the cosine distances among samples but changes the Euclidean distances among samples, and then it would disrupt the discriminative information extracted by dimensional reduction. In this paper, we design a feature transformation named SPPDAFT to reduce the gap between the dimensional reduction and SRC.

\section{B. SPPDAFT}

Given $n$ training samples $X=\left[x_{1}, x_{2}, \cdots x_{n}\right] \in \mathrm{R}^{m \times n}$, and a testing sample $x \in \mathrm{R}^{m}$, where $m$ is the dimension of the sample, $n$ is the number of the training samples. The SPPDAFT $x_{i} \in \mathrm{R}^{m} \rightarrow y_{i} \in \mathrm{R}^{n}$ for a training sample can be defined as:

$$
y_{i}=\left[d_{s}\left(x_{i}, x_{1}\right), d_{s}\left(x_{i}, x_{2}\right), \cdots, d_{s}\left(x_{i}, x_{n}\right)\right]^{T}
$$

The SPPDAFT $x_{i} \in \mathrm{R}^{m} \rightarrow y_{i} \in \mathrm{R}^{n}$ for a training sample can be defined as:

$$
y=\left[d_{s}\left(x, x_{1}\right), d_{s}\left(x, x_{2}\right), \cdots, d_{s}\left(x, x_{n}\right)\right]^{T}
$$

Where $d_{s}\left(x_{i}, x_{j}\right)$ is defined as:

$$
d_{s}\left(x_{i}, x_{j}\right)=\exp \left(-w_{i j}^{2}\right), w_{i j}=\sqrt{d_{E}^{2}\left(x_{i}, x_{j}\right) / \sigma_{i} \sigma_{j}}
$$

Where $\sigma_{i}=d_{E}\left(x_{i}, x_{i}^{k}\right)$ and $\sigma_{j}=d_{E}\left(x_{j}, x_{j}^{k}\right)$ are two local scale parameters, $x_{i}^{k}$ and $x_{j}^{k}$ are the $k$-th neighbor of $x_{i}$ and $x_{j}$, and $d_{E}\left(x_{i}, x_{j}\right)$ is the Euclidean distance between $x_{i}$ and $x_{j}$. It can be seen from Eq.(7) and (8) that SPPDAFT transforms the features to a new feature space, where the $j$-th feature of $y_{i}$ is $d_{s}\left(x_{i}, x_{j}\right)$. To describe simply, $\mathrm{R}^{m}$ is marked as low dimension space and $\mathrm{R}^{n}$ is marked as SPPD space.

Obviously, if cosine distances in SPPD space can increase with the Euclidean distances in low dimension space, the gap between dimensional reduction and SRC can be reduced. The cosine distance between two samples in SPPD space can be computed by Eq.(10). To analyze the attribution of $d_{c}\left(y_{i}, y_{j}\right)$, we firstly analyze the attributions of $y_{i}^{T} y_{j}$ and $\left|y_{i}\right|$.

$$
d_{c}\left(y_{i}, y_{j}\right)=1-y_{i}^{T} y_{j} /\left(\left|y_{i}\right|\left|y_{j}\right|\right)
$$

Proposition 1: $y_{i}{ }^{T} y_{j}$ decreases with the increase of $d_{E}\left(x_{i}, x_{j}\right)$.

Prove: According to Eq.(7) and (8), $y_{i}^{T} y_{j}$ can be rewritten as:

$$
\begin{aligned}
& y_{i}^{T} y_{j}=\sum_{s=1}^{n} y_{i s} * y_{j s} \\
& =\sum_{s=1}^{n} \exp \left(-d_{E}^{2}\left(x_{i}, x_{s}\right) / \sigma_{i} \sigma_{s} * \exp \left(-d_{E}^{2}\left(x_{j}, x_{s}\right) / \sigma_{j} \sigma_{s}\right.\right. \\
& =\sum_{s=1}^{n} e^{\left(-d_{E}^{2}\left(x_{i}, x_{s}\right) / \sigma_{i} \sigma_{j}\right)^{*}\left(\sigma_{j} / \sigma_{s}\right)} * e^{\left(-d_{E}^{2}\left(x_{j}, x_{s}\right) / \sigma_{i} \sigma_{j}\right) *\left(\sigma_{i} / \sigma_{s}\right)}
\end{aligned}
$$

If the samples are uniformly distributed in the feature space, $\sigma_{i}, \sigma_{s}, \sigma_{j}$ are equal, and then $\sigma_{i} / \sigma_{s}=1$ and $\sigma_{j} \sigma_{s}=1$, as a result, $y_{i}^{T} y_{j}$ can be seen as the joint probability distribution function of two Gaussian distributions that are only with different means. Obviously, the smaller the absolute of subtraction of the means of two Gaussian distributions, the bigger the $y_{i}^{T} y_{j}$, which means that $y_{i}^{T} y_{j}$ decreases with the increase of $d_{E}\left(x_{i}, x_{j}\right)$.

If the samples are not uniformly distributed in feature space, $y_{i}^{T} y_{j}$ can be seen as the joint probability distribution function of two Gaussian distributions weighted by $\sigma_{i} / \sigma_{s}$ and $\sigma_{j} / \sigma_{s}$. Furthermore, for the given $x_{i}, x_{j}$ and $X=\left[x_{1}, x_{2}, \cdots x_{n}\right] \in \mathrm{R}^{m \times n}$, $\sigma_{i} / \sigma_{s}$ and $\sigma_{j} / \sigma_{s}$ are two positive constants. As a result, $y_{i}^{T} y_{j}$ also decreases with the increase of $d_{\varepsilon}\left(x_{i}, x_{j}\right)$.

(a) $d s(x i, x s)$

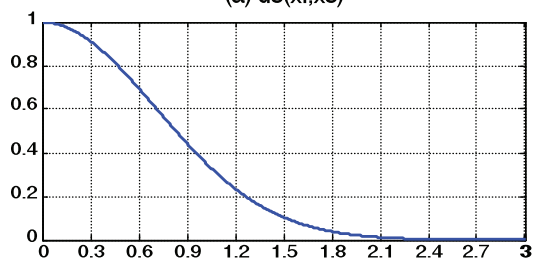

(b) derivative of $d s(x i, x s)$

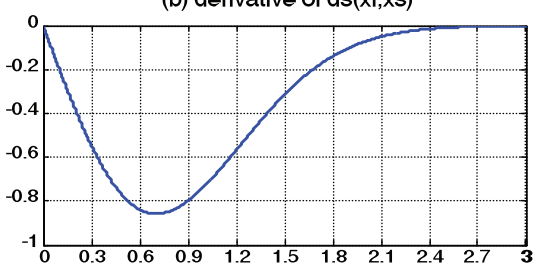

Fig.2 $d_{s}\left(x_{i}, x_{s}\right)$ and its derivative, where $x$ - axis is $w_{i j}$ 
Proposition 2: $\left|y_{1}\right| \approx\left|y_{2}\right| \approx \cdots \approx\left|y_{n}\right|$, when we set suitable values for $\sigma_{i}, i=1,2, \cdots, n$.

Prove: According to Eq.(7) and (8), $\left|y_{i}\right|$ can be rewritten as eq.(12). The function curve of $d_{s}\left(x_{i}, x_{s}\right)$ is presented in Fig.2. It can be seen from Fig. 2 that $d_{s}\left(x_{i}, x_{s}\right)$ owns three attributions shown in Eq.(13). Obviously, if we can make that the number of $d_{s}\left(x_{i}, x_{s}\right)$ of $y_{1}, y_{2}, \cdots, y_{n}$ in each interval are nearly the same, $\left|y_{1}\right| \approx\left|y_{2}\right| \approx \cdots \approx\left|y_{n}\right|$.

$$
\begin{aligned}
& \left|y_{i}\right|=\sqrt{\sum_{s=1}^{n} y_{i s} * y_{i s}}=\sqrt{\sum_{s=1}^{n} d_{s}\left(x_{i}, x_{s}\right)^{2}} \\
& \left\{\begin{array}{l}
d_{s}\left(x_{i}, x_{s}\right) \text { is large, when } w_{i s}<=0.3 \\
d_{s}\left(x_{i}, x_{s}\right) \text { decreases quickly, when } 0.3<w_{i s}<1.2 \\
d_{s}\left(x_{i}, x_{s}\right) \text { is small when } w_{i s}>=1.2
\end{array}\right.
\end{aligned}
$$

Now we turn to make the number of $d_{s}\left(x_{i}, x_{s}\right)$ of $y_{1}, y_{2}, \cdots, y_{n}$ in each interval nearly the same. For the training samples, because the goal of most dimensional reduction methods is maximizing the Euclidean distances across classes and minimizing the Euclidean distances within the class, the Euclidean distances within the class are often much smaller than the Euclidean distances across classes, and then if setting $k=2 * n_{c}$ in computing $\sigma_{i}, d_{E}\left(x_{i}, x_{i}^{n c}\right)$ would be much smaller than $d_{E}\left(x_{i}, x_{i}^{k}\right) / 2$, where $n_{c}$ is the number of training points in $c$-th class. As a result, $w_{i s}$ has three attributions shown in Eq.(14), which means that the number of $d_{s}\left(x_{i}, x_{s}\right)$ of $y_{1}, y_{2}, \cdots, y_{n}$ in each interval are nearly the same.

$$
\left\{\begin{array}{l}
w_{i s}<=0.3, \text { when } x_{s} \in N^{k / 2}\left(x_{i}\right) \\
0.3<w_{i s}<1.0, \text { when } x_{s} \notin N^{k / 2}\left(x_{i}\right) \text { and } x_{s} \in N^{k}\left(x_{i}\right) \\
w_{i s}>=1.0, \text { when } x_{s} \notin N^{k}\left(x_{i}\right)
\end{array}\right.
$$

It can be seen from proposition 1 and proposition 2 that $d_{c}\left(y_{i}, y_{j}\right)$ nearly increase with $d_{E}\left(x_{i}, x_{j}\right)$, which means that the cosine distances in SPPD space increase with the Euclidean distances in low dimension space, and then the gap between dimensional reduction and SRC can be reduced by SPPDAFT.

C. The algorithm of Adaptive Feature Transformation for Classification with Sparse Representation (SPPDSRC)

SPPDAFT is performed before SRC and after dimensional reduction, and the algorithm of SPPDAFT for classification with sparse representation (SPPDSRC) can be concluded as follow:

\section{Algorithm 1 SPPDSRC}

Input: labeled samples $\left\{z_{1}, z_{2}, \cdots, z_{n}\right\} \in \mathrm{R}^{h}$, the labels of labeled data $\left\{l_{1}, l_{2}, \cdots l_{n}\right\}$, a testing sample $z$, the mapping matrix $W$ obtained by a dimensional reduction method.

Output: the label $l$ of $z$.

Algorithm:

\section{//Initialization}

1 : set $k=2 * n_{c}$, where $n_{c}$ is the number of training samples in $c$-th class.

//Dimensional Reduction:

2: Map $\left\{z_{1}, z_{2}, \cdots z_{n}, z\right\} \in \mathrm{R}^{h}$ to $\left\{x_{1}, x_{2}, \cdots x_{n}, x\right\} \in \mathrm{R}^{m}$ by $W$, where $m$ is the dimension of features in low dimensional space.

\section{// SPPDAFT:}

3: Transform $\left\{x_{1}, x_{2}, \cdots x_{n}\right\} \in \mathrm{R}^{m} \rightarrow\left\{y_{1}, y_{2}, \cdots y_{n}\right\} \in \mathrm{R}^{n}$ by Eq.(7), and transform $x \in \mathrm{R}^{m} \rightarrow y \in \mathrm{R}^{n}$ by Eq. (8).

//Traditional Sparse Representation Classification:

4: Set $D=\left[y_{1}, y_{2}, \cdots y_{n}\right]$ and set the testing point $x=y$.

5: Compute $a$ by optimizing Eq. (1).

6: Compute $E^{k}$ for each class by Eq. (2), and get label $l$ of $x$ by Eq.(3)

\section{Compared with related algorithms}

Although both SPPDSRC and KSRC [6, 7] transform the features to a new feature space before classification, SPPDSRC is different with KSRC. In KSRC, the parameters of kernel functions such as RBF kernel and polynomial kernel are constants, however, the densities of samples are often different, so that found a good kernel parameter for all samples is very hard or impossible. However, in SPPDSRC, the parameters $\sigma_{i}$ and $\sigma_{j}$ of SPPDAFT are changed with the densities of $x_{i}$ and $x_{j}$, so that SPPDSRC is more adapted to the variability of data. Furthermore, in KSRC, the samples are normalized to have unit $l_{2}$-norm, and then some discriminative information extracted by dimensional reduction could be disrupted. However, in SPPDSRC, the samples need not be normalized to have unit $l_{2}$-norm. Obviously, SPPDSRC can utilize more discriminative information extracted by dimensional reduction.

Although both SPPDSRC and dictionary learning methods such as LCKSVD [9] and FDDL [13] can exploit the discriminative information hidden in the training samples, SPPDSRC is different with these dictionary learning methods. Firstly, in LCKSVD and FDDL, some constraints are added on the sparse coefficients to learn the discriminative information; however, to learn the discriminative information for the sparse coefficients may be not really good for SRC. For example, given $D=\left[D_{1}, D_{2}, \cdots, D_{C}\right]$ and each $D_{i}$ has 9 bases, because the constraint of sparse coefficients, each sample could be well represented by only 3 bases, and then two samples in the same class may have much different sparse coefficients. However, in SPPDSRC, we directly make the dictionary containing discriminative information, which is a better way to learn the dictionary for SRC. Secondly, because the constraint of sparse coefficients, the coefficients would be greatly influenced by an unlabeled sample, and then the constraints added on coefficients are also changed sharply, as a result, the unlabeled samples are hardly be used in LCKSVD and FDDL. However, in SPPDSRC, the discriminative information is extracted by a dimensional reduction method, and then the unlabeled samples can be easily used in 
SPPDSRC. Thirdly, LCKSVD and FDDL use an iterative approach to learn the dictionary, so that the computational complexity of dictionary learning methods such as LCKSVD and FDDL are much higher than that of SPPDSRC.

\section{Experiments}

In this section, several benchmarks of face databases are used to verify the effectiveness of the proposed SPPDSRC, such as: AR database [47], ORL database [48], Yale database [49], and CMU Multi-PIE database [50]. Following algorithms are used to compare: (1) the algorithms that employ dimensional reduction under the SRC framework, including SRC with LRGPSSDR [44], DSNPE [30], and LDA [45]. (2) The algorithms that employ LRGPSSDR under the KSRC framework, where three kernels are unitized such as: linear kernel, polynomial kernel, and RBF kernel. (3) The algorithms employ dictionary learning under the SRC framework, including LCKSVD [9], DLLR [12], and FDDL [13]. The SPAMS package or GPSR [34] can be used to solve Eq.(1), and the results of them are nearly the same, in this paper, we use SPAMS package to solve Eq.(1), where $\varepsilon$ is set to $10^{-4}$. All samples for SRC are normalized with length 1, because SRC gets a better result when the samples are normalized with length 1. The parameter of polynomial kernel is set to 3 . The parameter of RBF kernel is set by the median value of $1 /\left(\left\|x_{i}-\bar{x}\right\|^{2}\right), i=1, \cdots, n, \bar{x}$ is the mean of all training samples. The dimension of subspace of LRGPSSDR is 10 to 140 , where the interval is 20 .

For all databases, 2 6 samples per subject are randomly chose for labeled training samples, and 3 samples per subject are randomly chose for unlabeled training samples, while the other samples were used as query samples, The experiments were repeated 30 times to calculate the average recognition rate and the corresponding standard deviation. The accuracy is utilized to evaluate the experiments.

\section{A. Face recognition}

AR database [47]. The AR database consists of over 4,000 frontal images from 126 individuals. For each individual, 26 pictures were taken in two separate sessions. In our experiments, a subset that contains 50 males and 50 females with 6 illumination and 8 expression variations in two sessions is used. All images are resized to $32 \times 32$. The accuracies of competing methods are listed in Table 1 and the accuracies of SRC and SPPDSRC with LRGPSSDR, DSNPE, and LDA are listed in Fig.3.

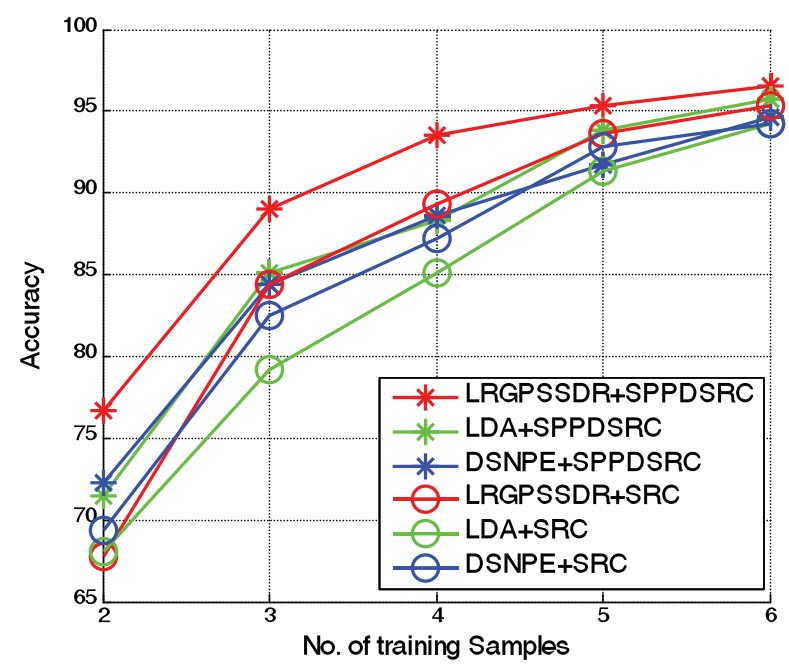

Fig.3 Experiments on AR with different number of training samples.

From the experiments on AR database, we can get four concludes. (1) It can be seen from Fig.3 that most of accuracies of SPPDSRC with dimensional reduction methods are higher than the accuracies of SRC with dimensional reduction, it proves that reducing the gap between dimensional reduction and SRC by SPPDAFT is very important. (2) It can be seen from Table 1 that the accuracies of LRGPSSDR+SPPDSRC are higher than the accuracies of LRGPSSDR+KSRC, and the accuracies of LRGPSSDR+KSRC are higher than the accuracies of LRGPSSDR+SRC, it proves that SPPDSRC is better than KSRC and KSRC is better than SRC on ORL. (3) It can be seen from Table 1 that most of accuracies of LDA+SPPDSRC are higher than the accuracies of DLLR, FDDL, and LCKSVD; it proves that the reconstruction ability of each basis should be changed reasonably in learning dictionary. (4) It can be seen from Table 1 that the accuracies of LRGPSSDR+SPPDSRC are much higher than the accuracies of FDDL and LCKSVD; it proves that the unlabeled samples are very important for sparse representation classification; however, the unlabeled samples are hardly used in dictionary learning methods such as FDDL and LCKSVD.

TABLE 1

Experiments by using all compared algorithms on AR with different number of training samples.

\begin{tabular}{cccccc}
\hline \hline No. of training Samples & 2 & 3 & 4 & 5 & 6 \\
\hline LRGPSSDR+SPPDSRC & $\mathbf{7 6 . 6 6} \pm \mathbf{2 . 4 0}$ & $\mathbf{8 9 . 0 3} \pm \mathbf{1 . 3 7}$ & $\mathbf{9 3 . 5 7} \pm \mathbf{0 . 9 7}$ & $\mathbf{9 5 . 3 3} \pm \mathbf{0 . 9 0}$ & $\mathbf{9 6 . 5 4} \pm \mathbf{0 . 8 4}$ \\
LDA+SPPDSRC & $71.47 \pm 1.66$ & $85.12 \pm 1.35$ & $88.35 \pm 1.19$ & $93.83 \pm 0.84$ & $95.74 \pm 0.95$ \\
DSNPE+SPPDSRC & $72.31 \pm 1.79$ & $84.42 \pm 1.25$ & $88.61 \pm 1.27$ & $91.73 \pm 1.09$ & $94.62 \pm 1.13$ \\
\hline LRGPSSDR+SRC & $67.80 \pm 3.10$ & $84.43 \pm 1.84$ & $89.35 \pm 1.59$ & $93.60 \pm 1.04$ & $95.38 \pm 1.10$ \\
LDA+SRC & $68.02 \pm 1.98$ & $79.19 \pm 1.56$ & $85.09 \pm 1.34$ & $91.37 \pm 1.20$ & $94.20 \pm 1.26$ \\
DSNPE+SRC & $69.36 \pm 2.11$ & $82.53 \pm 1.63$ & $87.21 \pm 1.55$ & $92.85 \pm 1.17$ & $94.20 \pm 1.30$ \\
\hline LRGPSSDR+KSRC(Linear) & $70.05 \pm 2.30$ & $86.67 \pm 1.41$ & $91.32 \pm 1.08$ & $94.35 \pm 1.09$ & $95.60 \pm 0.87$ \\
LRGPSSDR+KSRC(Polynomial) & $74.04 \pm 4.15$ & $88.38 \pm 1.19$ & $92.19 \pm 1.33$ & $94.02 \pm 0.62$ & $96.21 \pm 1.34$ \\
LRGPSSDR+KSRC(RBF) & $74.89 \pm 2.13$ & $88.44 \pm 1.16$ & $92.63 \pm 0.98$ & $94.46 \pm 0.63$ & $96.31 \pm 0.94$ \\
\hline DLLR [12, 2014] & $73.40 \pm 3.70$ & $78.39 \pm 1.83$ & $85.20 \pm 1.35$ & $90.78 \pm 1.07$ & $92.65 \pm 1.15$ \\
FDDL[13, 2011] & $67.80 \pm 1.54$ & $73.09 \pm 1.90$ & $87.46 \pm 1.60$ & $92.66 \pm 1.31$ & $94.07 \pm 1.42$
\end{tabular}




\begin{tabular}{llllll}
$\operatorname{LCKSVD}[9,2013]$ & $74.00 \pm 1.58$ & $81.69 \pm 0.88$ & $86.26 \pm 1.37$ & $91.67 \pm 0.80$ & $93.32 \pm 0.93$ \\
\hline \hline
\end{tabular}

TABLE 2

Experiments by using all compared algorithms on ORL with different number of training samples.

\begin{tabular}{cccccc}
\hline \hline No. of training Samples & 2 & 3 & 4 & 5 & 6 \\
\hline LRGPSSDR+SPPDSRC & $\mathbf{8 6 . 8 2} \pm \mathbf{2 . 5 6}$ & $\mathbf{9 3 . 3 1} \pm \mathbf{2 . 5 9}$ & $\mathbf{9 6 . 3 8} \pm \mathbf{2 . 0 3}$ & $\mathbf{9 7 . 8 1} \pm \mathbf{1 . 5 6}$ & $\mathbf{9 8 . 6 3} \pm \mathbf{1 . 3 2}$ \\
LDA+SPPDSRC & $80.95 \pm 2.97$ & $87.90 \pm 1.84$ & $91.83 \pm 2.22$ & $94.25 \pm 3.02$ & $94.00 \pm 3.18$ \\
DSNPE+SPPDSRC & $83.73 \pm 2.66$ & $89.62 \pm 2.03$ & $93.22 \pm 2.35$ & $95.72 \pm 2.89$ & $96.33 \pm 2.45$ \\
\hline LRGPSSDR+SRC & $84.45 \pm 2.38$ & $91.38 \pm 2.67$ & $95.58 \pm 2.13$ & $97.18 \pm 2.29$ & $98.25 \pm 1.54$ \\
LDA+SRC & $79.15 \pm 3.22$ & $86.31 \pm 2.95$ & $90.00 \pm 2.51$ & $93.00 \pm 3.13$ & $93.65 \pm 3.49$ \\
DSNPE+SRC & $81.25 \pm 2.58$ & $88.53 \pm 2.29$ & $92.73 \pm 2.17$ & $94.66 \pm 2.79$ & $95.72 \pm 2.58$ \\
\hline LRGPSSDR+KSRC(Linear) & $81.70 \pm 2.84$ & $90.50 \pm 2.45$ & $95.08 \pm 1.18$ & $96.88 \pm 1.79$ & $97.75 \pm 1.97$ \\
LRGPSSDR+KSRC(Polynomial) & $77.95 \pm 5.49$ & $86.44 \pm 5.71$ & $91.17 \pm 3.73$ & $96.88 \pm 2.14$ & $95.00 \pm 2.64$ \\
LRGPSSDR+KSRC(RBF) & $85.35 \pm 2.98$ & $91.93 \pm 1.86$ & $95.62 \pm 1.19$ & $96.88 \pm 1.69$ & $98.25 \pm 1.54$ \\
\hline DLLR [12,2014] & $73.40 \pm 3.70$ & $86.26 \pm 2.74$ & $89.76 \pm 2.12$ & $91.36 \pm 3.23$ & $92.08 \pm 2.97$ \\
FDDL[13,2011] & $76.48 \pm 3.24$ & $82.28 \pm 3.35$ & $91.33 \pm 2.74$ & $93.36 \pm 2.66$ & $94.53 \pm 2.35$ \\
LCKSVD[9,2013] & $81.90 \pm 2.62$ & $88.25 \pm 2.99$ & $91.50 \pm 2.22$ & $92.87 \pm 1.89$ & $93.75 \pm 1.76$ \\
\hline \hline
\end{tabular}

TABLE 3

Experiments by using all compared algorithms on Yale with different number of training samples.

\begin{tabular}{cccccc}
\hline \hline No. of training Samples & 2 & 3 & 4 & 5 & 6 \\
\hline LRGPSSDR+ SPPDSRC & $60.67 \pm 4.88$ & $\mathbf{7 3 . 5 3} \pm \mathbf{4 . 9 0}$ & $\mathbf{7 9 . 5 0} \pm \mathbf{4 . 5 0}$ & $\mathbf{8 4 . 0 0} \pm \mathbf{4 . 8 1}$ & $84.50 \pm 4.87$ \\
LDA+ SPPDSRC & $61.00 \pm 6.68$ & $71.13 \pm 4.18$ & $78.58 \pm 4.37$ & $79.78 \pm 4.61$ & $82.33 \pm 6.84$ \\
DSNPE + SPPDSRC & $\mathbf{6 2 . 5 3} \pm \mathbf{6 . 6 8}$ & $71.93 \pm 5.62$ & $77.50 \pm 5.11$ & $80.55 \pm 5.21$ & $83.67 \pm 6.18$ \\
\hline LRGPSSDR+SRC & $55.78 \pm 4.49$ & $65.53 \pm 5.33$ & $72.42 \pm 5.76$ & $77.11 \pm 4.97$ & $79.83 \pm 5.75$ \\
LDA+SRC & $56.11 \pm 4.80$ & $66.93 \pm 4.53$ & $72.67 \pm 5.68$ & $74.67 \pm 6.01$ & $78.83 \pm 6.60$ \\
DSNPE+SRC & $58.89 \pm 4.75$ & $68.33 \pm 5.17$ & $73.67 \pm 4.96$ & $75.44 \pm 5.97$ & $79.22 \pm 4.40$ \\
\hline LRGPSSDR+KSRC(Linear) & $53.44 \pm 6.22$ & $64.53 \pm 3.74$ & $71.67 \pm 5.82$ & $75.89 \pm 5.97$ & $79.00 \pm 4.49$ \\
LRGPSSDR+KSRC(Polynomial) & $46.33 \pm 5.34$ & $56.53 \pm 5.52$ & $59.67 \pm 6.13$ & $82.33 \pm 4.47$ & $75.33 \pm 6.12$ \\
LRGPSSDR+KSRC(RBF) & $62.00 \pm 5.72$ & $72.33 \pm 4.37$ & $78.50 \pm 5.22$ & $81.78 \pm 6.60$ & $\mathbf{8 4 . 6 7} \pm \mathbf{3 . 3 1}$ \\
\hline DLLR [12,2014] & $58.78 \pm 5.19$ & $67.60 \pm 4.57$ & $75.58 \pm 4.37$ & $78.66 \pm 4.86$ & $79.54 \pm 4.03$ \\
FDDL[13,2011] & $52.56 \pm 4.74$ & $64.80 \pm 5.61$ & $77.26 \pm 4.70$ & $81.69 \pm 5.21$ & $81.67 \pm 6.08$ \\
LCKSVD[9, 2013] & $62.06 \pm 5.82$ & $69.33 \pm 5.22$ & $76.17 \pm 5.39$ & $79.86 \pm 3.89$ & $80.61 \pm 4.29$ \\
\hline \hline
\end{tabular}

ORL database [48]. The ORL database consists of 400 frontal images from 40 individuals, all images are resized to $32 \times 32$. The accuracies of competing methods are listed in Table 2 and the accuracies of SRC and SPPDSRC with LRGPSSDR, DSNPE, and LDA are listed in Fig.4.

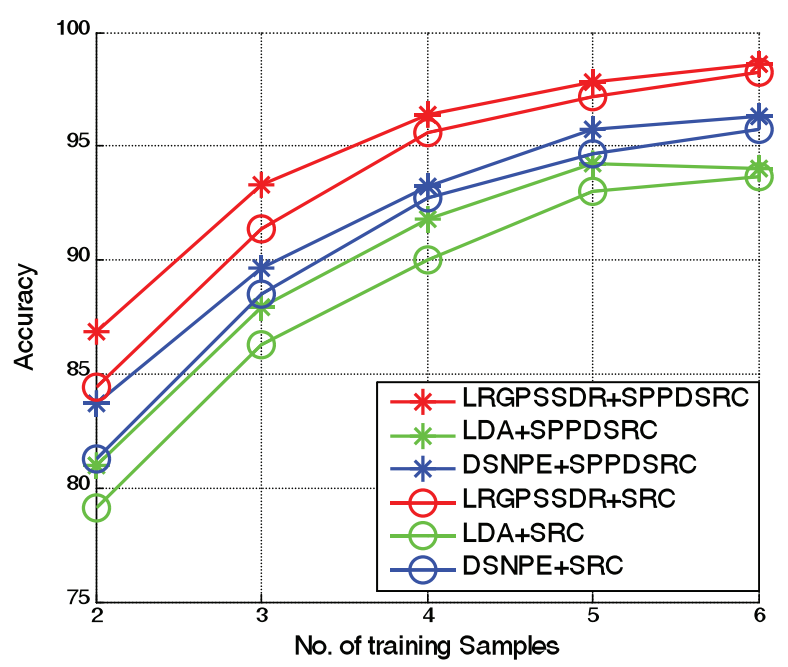

Fig.4 Experiments on the ORL with different number of training samples.

From the experiments on ORL database, we can get four similar concludes. (1) It can be seen from Fig.4 that most of accuracies of SPPDSRC with dimensional reduction are also higher than the accuracies of SRC with dimensional reduction, it proves that reducing the gap between SRC and dimensional reduction by SPPDAFT is also very important on ORL. (2) It can be seen from Table 2 that the accuracies of LRGPSSDR+SPPDSRC are also higher than the accuracies of LRGPSSDR+KSRC obviously, SPPDSRC is also better than KSRC on ORL. (3) It can be seen from Table 2 that most of accuracies of LDA+SPPDSRC are also higher than the accuracies of DLLR, FDDL, and LCKSVD; it proves that adding constraints for the sparse coefficients cannot learn good discriminative information for dictionary on ORL. (4) It can be seen from Table 2 that the accuracies of LRGPSSDR+ SPPDSRC are also much higher than the accuracies of DLLR and LCKSVD.

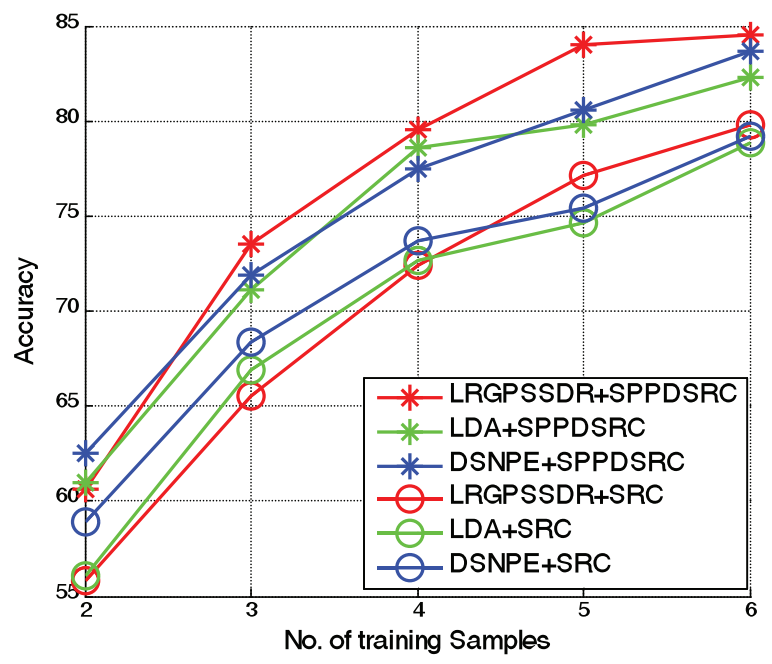


Fig.5 Experiments on the Yale with different number of training samples.

Yale database [49]. The Yale database consists of 165 frontal images from 15 individuals, and all images are resized to $32 \times 32$. The accuracies of competing methods are listed in Table 3 and the accuracies of SRC and SPPDSRC with LRGPSSDR, DSNPE, and LDA are listed in Fig.5. It can be seen from Table. 3 and Fig.5 that four similar concludes are also existed on Yale. However, the accuracies of DSNPE+ SPPDSRC are higher than the accuracies of LRGPSSDR+ SPPDSRC when the number of labeled training sample is set to 2; we think the reason is that the assumed manifold in LRGPSSDR may be not suitable for this database.

CMU Multi-PIE database[50]. The PIE database contains 41368 images of 68 individuals, each person under 13 different poses, 43 different illumination conditions, and with 4 different expressions. In this paper, we only use five near frontal poses and all the images under different illuminations and expressions, all images are resized to $32 \times 32$. The accuracies of competing methods are listed in Table 4 and the accuracies of SRC and SPPDSRC with LRGPSSDR, DSNPE, and LDA are listed in Fig.6. It can be seen from Table.4 and Fig. 6 that four similar concludes are also existed on PIE.

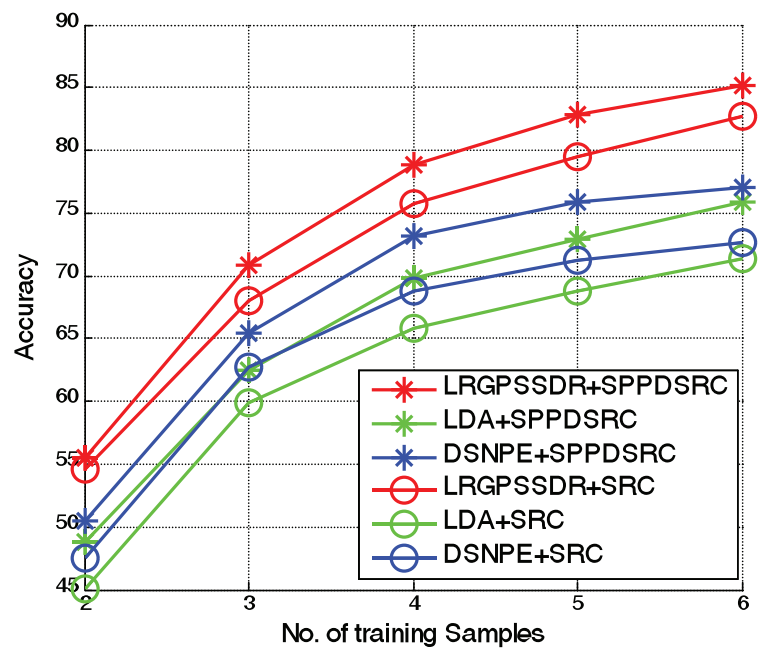

Fig.6 Experiments on PIE with different number of training samples.

TABLE 4

Experiments by using all compared algorithms on PIE with different number of training samples.

\begin{tabular}{cccccc}
\hline \hline No. of training Samples & 2 & 3 & 4 & 5 & 6 \\
\hline LRGPSSDR+SPPDSRC & $\mathbf{5 5 . 4 7} \pm \mathbf{2 . 7 7}$ & $\mathbf{7 0 . 9 1} \pm \mathbf{3 . 4 7}$ & $\mathbf{7 8 . 8 2} \pm \mathbf{2 . 8 5}$ & $\mathbf{8 2 . 8 4} \pm \mathbf{3 . 0 9}$ & $\mathbf{8 5 . 1 5} \pm \mathbf{2 . 8 5}$ \\
LDA+SPPDSRC & $48.75 \pm 2.88$ & $62.51 \pm 3.71$ & $69.78 \pm 2.90$ & $72.97 \pm 3.14$ & $75.86 \pm 2.71$ \\
DSNPE+SPPDSRC & $50.47 \pm 3.26$ & $65.49 \pm 3.44$ & $73.18 \pm 3.12$ & $75.92 \pm 2.90$ & $77.06 \pm 3.00$ \\
\hline LRGPSSDR+SRC & $54.56 \pm 2.99$ & $68.01 \pm 3.21$ & $75.69 \pm 3.11$ & $79.46 \pm 2.80$ & $82.67 \pm 2.96$ \\
LDA+SRC & $45.12 \pm 3.11$ & $59.88 \pm 3.86$ & $65.86 \pm 2.94$ & $68.80 \pm 3.81$ & $71.35 \pm 2.35$ \\
DSNPE+SRC & $47.57 \pm 3.23$ & $62.71 \pm 3.75$ & $68.77 \pm 2.52$ & $71.25 \pm 2.40$ & $72.70 \pm 2.01$ \\
\hline LRGPSSDR+KSRC(Linear) & $51.55 \pm 4.51$ & $67.53 \pm 3.32$ & $74.75 \pm 3.13$ & $78.67 \pm 2.74$ & $81.57 \pm 2.98$ \\
LRGPSSDR+KSRC(Polynomial) & $47.92 \pm 3.49$ & $60.52 \pm 4.46$ & $64.56 \pm 3.19$ & $67.65 \pm 5.15$ & $66.72 \pm 6.14$ \\
LRGPSSDR+KSRC(RBF) & $55.13 \pm 4.76$ & $69.50 \pm 3.66$ & $77.84 \pm 2.24$ & $81.55 \pm 2.03$ & $84.11 \pm 2.95$ \\
\hline DLLR [12,2014] & $47.40 \pm 3.67$ & $66.18 \pm 3.52$ & $69.68 \pm 2.67$ & $71.17 \pm 3.01$ & $74.38 \pm 3.40$ \\
FDDL[13,2011] & $48.48 \pm 2.82$ & $65.49 \pm 3.08$ & $71.75 \pm 2.71$ & $75.21 \pm 2.84$ & $79.52 \pm 3.13$ \\
LCKSVD[9,2013] & $47.38 \pm 3.07$ & $57.16 \pm 3.21$ & $68.39 \pm 3.56$ & $74.68 \pm 2.84$ & $78.85 \pm 2.84$ \\
\hline \hline
\end{tabular}

TABLE 5

Averaged training time of experiments that with different number of training samples on $\operatorname{AR}(s)$

\begin{tabular}{cccccc}
\hline \hline No. of training Samples & 2 & 3 & 4 & 5 & 6 \\
\hline LRGPSSDR & 0.2538 & 0.4358 & 0.5047 & 0.5549 & 0.7913 \\
LDA & 0.0335 & 0.0605 & 0.0757 & 0.0882 & 0.1011 \\
DSNPE & 0.1625 & 0.4123 & 0.6639 & 1.004 & 1.520 \\
DLLR & 2.864 & 3.724 & 5.430 & 11.53 & 20.46 \\
FDDL & 146.5 & 382.4 & 834.3 & 1682 & 2337 \\
LCKSVD & 9.975 & 13.26 & 20.16 & 34.06 & 46.71 \\
\hline \hline
\end{tabular}

\section{B. Computational time}

In this subsection, we present the averaged training time of all algorithms, the runtime estimates reported in this paper are obtained on an Intel Core i5-3470 PC with four $3.2 \mathrm{GHz}$ processors and 8GB RAM. Obviously, the running time will be greatly increased if these algorithms are performed on an embedded system which is the operating environment of most face recognition system.

In training processing, for LRGPSSDR+SPPDSRC, LDA+SPPDSRC, DSNPE+ SPPDSRC, LRGPSSDR+SRC, LDA+SRC, DSNPE+SRC, LRGPSSDR+KSRC(Linear), RGPSSDR+KSRC(Polynomial), LRGPSSDR+ KSRC(RBF), only the training processing of the corresponding dimensional reduction algorithm need to be performed; for FDDL, DLLR, and LCKSVD, dictionary learning need to be performed. The averaged training time on AR with different number of training samples are presented in Table.5, the averaged training time on other databases have the similar laws. It can be seen from the Table.5 that the averaged training time of FDDL DLLR, LCKSVD are significantly longer than that of LRGPSSDR, LDA, and DSNPE; especially, the training time of FDDL is 2337 seconds when 6 labeled training samples per subject are used in training. However, the training time of most face recognition system cannot be too long, for the reason that we may frequently add a person to or remove a person from the face recognition system, which makes that the training processing may be frequently performed. As a result, our algorithm is more important than dictionary learning methods when the face recognition system runs in an 
embedded system or the face recognition system is used to recognize a large number of persons.

\section{Conclusion}

In this paper, an adaptive feature transformation named SPPDAFT is proposed; SPPDAFT transforms features to a new feature space, and makes that the cosine distances among samples in new feature space increase with the Euclidean distances among samples in original space. As a result, the dictionary in new feature space contains the discriminative information extracted by dimensional reduction methods, and then the gap between dimensional reduction and SRC can be reduced. The experiments on four face databases prove that our method is greatly improved SRC, and better than the KSRC and some dictionary learning methods such as FDDL DLLR, LCKSVD.

Besides the cosine distance among bases and a testing sample influence the reconstruction ability of these bases to this testing sample, the positional relationships among bases and this testing sample are also influence the reconstruction ability of these bases to this testing sample, for example, in LARs algorithm, if the bases are symmetry with each other and the symmetry axis is this testing sample, these bases would have good reconstruction ability to this testing sample, so that in the future we would design a new adaptive feature transformation that considers this case.

\section{ACKNOWLEDGMENT}

This work was supported by China National Science Foundation under Grants 60973083, 61273363, 61003174, State Key Laboratory of Brain and Cognitive Science under grants $08 \mathrm{~B} 12$.

\section{References}

[1] Haichao Zhang, Yanning Zhang, Thomas S. Huang, "Pose-robust face recognition via sparse representation," Pattern Recognition, vol. 46, no. 5, pp. 1511-1521, May. 2013.

[2] Congyan Lang, Bin cheng, Songhe Feng, Xiaotong Yuan, "Supervised sparse patch coding towards misalignment-robust face recognition," in Proc. International Conference on Image and Graphics (ICIG), HeiFei, 2011, pp. 12-15.

[3] Hua-wen Chang, Ming-hui Wang, Thomas S. Huang, "Sparse correlation coefficient for objective image quality assessment," Image Communication, vol. 26, no. 10, pp. 577-588, Nov. 2011.

[4] Cevher. V, Krause. A, "Face Recognition in Movie Trailers via Mean Sequence Sparse Representation-based Classification," in Proc. IEEE Conference on Computer Vision and Pattern Recognition (CVPR), Portland, 2013, pp. 3531-3538.

[5] Elhamifar. E, Vidal. R, "Sparse Subspace Clustering: Algorithm, Theory, and Applications," IEEE Transactions on Pattern Analysis and Machine Intelligence, vol. 35, no. 11, pp. 2765-2781, Nov. 2013.

[6] Li Zhang, Wei-Da Zhou, Fan-Zhang Li, "Kernel sparse representation-based classifier ensemble for face recognition," Multimedia Tools and Applications, vol. 117, pp. 50-60, Apr. 2013.

[7] Liu. J, Wu. Z, Wei. Z Xiao. L, "Spatial-Spectral Kernel Sparse Representation for Hyperspectral Image Classification," IEEE Journal of Selected Topics in Applied Earth Observations and Remote Sensing, vol. 6, no. 6, pp. 2462-2471, Dec. 2013.

[8] Raymond Ptucha, Andreas Savakis, "Manifold based sparse representation for facial understanding in natural images," Image and Vision computing, vol. 31, no. 5, pp. 365-378, May. 2013.

[9] Zhuolin Jiang, Zhe Lin, Davis, L.S., "Label Consistent K-SVD: Learning a Discriminative Dictionary for Recognition," IEEE
Transactions on Pattern Analysis and Machine Intelligence, vol. 35, no. 11, pp. 2651-2664, Nov. 2013.

[10] Haichao Zhang, Yanning Zhang, Thomas S. Huang, "Simultaneous discriminative projection and dictionary learning for sparse representation based classification," Pattern Recognition, vol. 46, no. 1, pp. 346-354, Jan. 2013

[11] Zhizhao Feng, Meng Yang, Lei Zhang, Yan Liu, David Zhang, "Joint Discriminative Dimensionality Reduction and Dictionary Learning for Face Recognition," in Proc. International Conference on Computer Vision and Pattern Recognition (CVPR), Columbus, 2014.

[12] Jie Chen, Zhang Yi, "Sparse representation for face recognition by discriminative low-rank matrix recovery," Journal of Visual Communication and Image Representation, vol. 25, no.5, pp. 763-773, Jul. 2014.

[13] Meng Yang, Lei Zhang, Xiangchu Feng, David Zhang, "Fisher Discrimination Dictionary Learning for Sparse Representation," in Proc. International Conference on Computer Vision (ICCV), Washington, 2011, pp. 543-550.

[14] Zhihui Lai, Wai Keung Wong, Zhong Jin, Jian Yang, Yong Xu, "Sparse Approximation to the Eigen subspace for Discrimination," IEEE Transactions on Neural Networks and Learning Systems, vol. 23, no. 12, pp. 1948-1960, Dec. 2012 .

[15] Baiyang Liu, Junzhou Huang, Kulikowski C, Lin Yang, "Robust Visual Tracking Using Local Sparse Appearance Model and K-Selection," IEEE Transactions on Pattern Analysis and Machine Intelligence, vol. 35, no. 12, pp. 2968-2981, Dec. 2013.

[16] Zhao Zhang, Shuicheng Yan, Mingbo Zhao, "Pairwise Sparsity Preserving Embedding for Unsupervised Subspace Learning and Classification," IEEE Transactions on Image Processing, vol. 22, no. 12, pp. 4640-4651, Dec. 2013.

[17] Weihua Ou, Xinge You, Yiu-ming Cheung, Qinmu Peng, "Structured Sparse Coding for Image Representation Based on L1-Graph," in Proc. International Conference on Pattern Recognition(ICPR), Tsukuba, 2012, pp. 3220-3223.

[18] Shenghua Gao, Ivor Wai-Hung Tsang, Liang-Tien Chia, "Laplacian Sparse Coding, Hypergraph Laplacian Sparse Coding, and Applications," IEEE Transactions on Pattern Analysis and Machine Intelligence, vol. 35, no. 1, pp. 92-104, Jan. 2013.

[19] J.Wang, J.Yang, K.Yu,F.Lv, T.Huang, Y.Gong, "Locality-constrained linear coding for image classification," in Proc. International Conference on Computer Vision and Pattern Recognition (CVPR), 2010, pp.3360-3367.

[20] M.Zheng, J.Bu, C.Chen, C.Wang, L.Zhang, G.Qiu, D.Cai, "Graph regularized sparse coding for image representation," IEEE Transactions on Image Processing, vol. 20, no. 5, pp. 1327-1336, May. 2011.

[21] Xiaoqiang Lu, Yuan Yuan, Pingkun Yan, "Robust visual tracking with discriminative sparse learning," Pattern Recognition, vol. 46, no. 7, pp. 1762-1771, Jul. 2013.

[22] Jim Jing-Yan Wang, Halima Bensmail, Xin Gao, "Discriminative sparse coding on multi-manifolds," Knowledge-Based Systems, vol. 54, pp. 199-206, Dec. 2013.

[23] Castrodad.A, Zhengming Xing, Greer.JB, Bosch.E, Carin, L, Sapiro.G, "Learning Discriminative Sparse Representations for Modeling, Source Separation, and Mapping of Hyperspectral Imagery," IEEE Geoscience and Remote Sensing Society, vol. 49, no. 11, pp. 4263-4281, Nov. 2011.

[24] Meng Jian, Cheolkon Jung, "Class-Discriminative Kernel Sparse Representation Based Classification Using Multi-Objective Optimization," IEEE Transactions on Signal Processing, vol. 61, no. 18, pp. 4416-4427, Sept. 2013.

[25] Meng Yang, Lei Zhang, Jian Yang, Zhang D, "Regularized Robust Coding for Face Recognition," IEEE Transactions on Image Processing, vol. 22, no. 5, pp. 1753-1766, May. 2013.

[26] Chia-Po Wei, Yu-WeiChao, Yi-RenYeh, Yu-Chiang Frank Wang, "Locality-sensitive dictionary learning for sparse representation based classification," Pattern Recognition, vol. 46, no. 5, pp. 1277-1287, May. 2013.

[27] Can-Yi Lu, Hai Min, Jie Gui, Lin Zhu, Ying-Ke Lei, "Face recognition via Weighted Sparse Representation," Journal of Visual Communication and Image Representation, vol. 24, no. 2, pp. 111-116, Feb. 2013.

[28] Wei Rao, Gang Li, Xiqin Wang, Xiang-Gen Xia , "Adaptive Sparse Recovery by Parametric Weighted L1 Minimization for ISAR Imaging of Uniformly Rotating Targets," IEEE Journal of Selected Topics in Applied Earth Observations and Remote Sensing, vol. 6, no. 6, pp. 942-952, Apr. 2013. 
[29] J. Mairal, F. Bach, J. Ponce, G. Sapiro, "Optimized projections for sparse representation based classification," Neurocomputing, vol. 113, no. 3, pp. 213-219, Aug. 2013.

[30] Jie Gui, Zhenan Sun, Wei Jia,Rongxiang Hu,Yingke Lei, Shuiwang Ji, "Discriminant sparse neighborhood preserving embedding for face recognition," Pattern Recognition, vol. 45, no. 8, pp. 2884-2893, Aug.2012.

[31] Lishan Qiao, Songcan Chen, Xiaoyang Tan, "Sparsity preserving projections with applications to face recognition," Pattern Recognition, vol. 43, no. 1, pp. 331-341, Jan.2010.

[32] Fei Yin, L.C. Jiao, Fanhua Shang, Lin Xiong, Xiaodong Wang, "Sparse regularization discriminant analysis for face recognition," Neurocomputing, vol. 128, no. 27, pp. 341-362, Mar. 2014.

[33] B. Efron, T. Hastie, I. Johnstone, R. Tibshirani, "Least angle regression," Annals of statistics, vol. 32, no. 2, pp. 407-499, 2004.

[34] $\mathrm{M}^{\prime}$ ario A. T. Figueiredo, Robert D. Nowak, Stephen J. Wright, "Gradient Projection for Sparse Reconstruction: Application to Compressed Sensing and Other Inverse Problems," IEEE Signal Processing Society, vol. 1, no. 4, pp. 586-597, Dec.2007.

[35] Lihi Zelnik-Manor, Pietro Perona , "Self-Tuning Spectral Clustering ," in Proc. Advances in Neural Information Processing Systems(NIPS), 2004, pp.1601-1608

[36] John Wright, Allen Y. Yang, Arvind Ganesh, S. Shankar Sastry, Yi Ma, "Robust Face Recognition via Sparse Representation," IEEE Transactions on Pattern Analysis and Machine Intelligence, vol. 31, no. 2, pp. 210-227, Feb. 2009.

[37] Xiaofei He, Deng Cai, Shuicheng Yan, Hong-Jiang Zhang, 'Neighborhood preserving embedding,' in Proc. International Conference on Computer Vision (ICCV), Beijing, 2005, pp. 1208-1213.

[38] Hong Huang,Jianwei Li,Jiamin Liu, "Enhanced semi-supervised local Fisher discriminant analysis for face recognition," Future Generation Computer Systems, vol. 28, no. 1, pp. 244-253, Jan. 2012.

[39] Mingbo Zhao, Zhao Zhang, Tommy W.S.Chow, "Trace ratio criterion based generalized discriminative learning for semi-supervised dimensionality reduction", Pattern Recognition, vol. 45, no. 4, pp. 1482-1499, Apr. 2012.

[40] Jun-Bao Li, Zhi-Ming Yang, Yang Yu, Zhen Sun, "Semi-supervised kernel learning based optical image recognition," Optics Communications, vol. 258, no. 18, pp. 3697-3703, Aug. 2012.

[41] B. Raducanu, F. Dornaika, "A supervised non-linear dimensionality reduction approach for manifold learning," Pattern Recognition, vol. 45, no. 6, pp. 2432-2444, Jun. 2012.

[42] Fanhua Shang, L.C. Jiao, Yuanyuan Liu, "Semi-supervised learning with nuclear norm regularization," Pattern Recognition, vol. 46, no. 8, pp. 2323-2336, Aug. 2013

[43] Qianying Wang, Pong C Yuen, Guocan Feng, "Semi-supervised metric learning via topology preserving multiple semi-supervised assumptions," Pattern Recognition, vol. 46, no. 9, pp. 2576-2578, Sept. 2013

[44] WEI Jia, PENG Hong, "Local and Global Preserving Based Semi-Supervised Dimensionality Reduction Method," Journal of Software, vol. 19, no. 11, pp. 2833-3842, Nov.2008

[45] Martinez AM, Kak AC, "PCA Versus LDA," IEEE Transactions on Pattern Analysis and Machine Intelligence, vol. 23, no. 2, pp. 228-233, Feb.2001

[46] Cover T, Hart P, "Nearest neighbor pattern classification," IEEE Transactions on Information Theory, vol. 13, no. 1, pp. 21-27, Jan. 1967.

[47] A.Martinez, R. Benavente, "The AR face database," CVC Technical Report 24, 1998.

[48] ORLface http://www.cl.cam.ac.uk/research/dtg/attarchive/facedatabase.html

[49] Yale Face database, http://vision.ucsd.edu/content/yale-face-database

[50] Sim T, Baker S, Bsat M, "The CMU Pose, Illumination, and Expression (PIE) database," Fifth IEEE International Conference on Automatic Face and Gesture Recognition, 2002, Washington, pp.46-51

Yaxin Sun is a Ph.D. candidate at the School of Computer Science and Engineering, South China University of Technology, Guangzhou, China. He received his M.Sc. degree in Computer Application Technology from Guangzhou University of Technology in 2012. His current research interests include speech emotion recognition, pattern recognition and machine learning.

Guihua Wen, born in 1968, Ph.D., professor, doctor supervisor. In 2005-2006, he did visiting research on machine learning and semantic web in School of Electronics and Computer, University of Southampton,UK. His main research interests are computational creativity, data mining and knowledge discovery, machine learning, and cognitive geometry. Since 2006, he proposed some original methods based on the computation of cognitive laws, which can effectively solve difficult problems in information science. The research results have been published in the international journals, including Pattern Recognition Neurocomputing, Journal of Software, Journal of computer Research and Development. He also published some papers in the international conferences such as IJCAI. Since 2006, he directed the projects from the China National Natural Science Foundation, State Key Laboratory of Brain and Cognitive Science, the Ministry of Education Scientific Research Foundation for returned overseas students, Guangdong Provincial Science and Technology research project, the Fundamental Research Funds for the Central Universities, SCUT. He also directed many projects from enterprises, with applications of his research results to the practical problems. He has ever been a Council Member of Chinese Association for Artificial Intelligence and a program committee member of many international conferences. He is also a reviewer for China National Natural Science Foundation. 